\title{
En una red de mundos plausibles. La urdimbre de la explicación ${ }^{3}$
}

\author{
J. FRANCISCO ÁLVAREZ \\ UNED, Madrid
}

En el estudio del complejo entramado de relaciones que puedan darse entre Filosofia y Literatura, en algún momento nos ha parecido interesante para ciertos problemas metodológicos el camino que va desde la Literatura hacia la Filosofia; camino que podía hacernos descubrir cómo cierto modelo de ser humano, que se pone en juego en algunas obras literarias (por ejemplo en Dostoievski), nos daba pie para refinar el individualismo metodológico y construir un modelo de individuo con mayor complejidad que el rational fool, el imbécil racional, de la teoría económica estándar. Aquí se intentarán dar algunos pasos que señalen las posibilidades de otro sentido del camino; ahora quizá podamos avanzar hacia el análisis literario, cuando no a la Literatura misma, desde la Filosofia. De hecho no estoy plenamente convencido del sentido de la relación. Desde luego se apuesta aqui por cierta temática propia de la Filosofía, se defiende cierto peculiar plano de la reflexión correspondiente a los instrumentos analíticos del conocimiento como específico del quehacer filosofico; pero las diferencias aparecen menos nitidas cuando la obra literaria se despliega en un plano autorreflexivo que incorpora como producto la materia misma de su producción. Quizá otro tanto cabe decir de algunos ensayos filosóficos en nuestra lengua, que han adquirido el papel de auténticas piezas literarias (mucho se ha hablado al respecto sobre el estilo en Ortega y Gasset o en María Zambrano). De he- cho es una cuestión abierta la de si hacer filosofía o hacer literatura vengan a ser asuntos similares o no. Posiblemente sea indispensable añadir otras orientaciones, otras direcciones que nos permitan multiplicar los sentidos, para al menos complicar el panorama, para hacerlo más difficil de ordenar. Variedad e incompletud del orden que más que vicio considero virtud intelectual.

Vengamos pues a trazar el mapa o al menos los majanos por donde van a discurrir algunas de nuestras sendas. Tres piezas son las que pretendemos mover y articular. La primera, un referente básico aquí, es ftalo Calvino; trataremos de jugar con su libro Si una noche de invierno un viajero, haciendo un uso directo de el, buscando su incorporación en la estructura narrativa del texto. La segunda es cierta crónica oral que escuché en México. La tercera pieza, donde aparecen más claramente las cuestiones gremialmente filosoficas, es cierto acopio de cuestiones metodologicas sobre historia de las ideas; propuestas metodológicas conectadas con los trabajos de algunos historiadores y filósofos de la Historia que tienen en común su relación con Cambridge: G.E.R. Lloyd, Quentin Skinner, John Dunn, A. Pagden y particularmente cl libro de G. Hawthorn, Plausible Worlds. Este último es un interesantísimo trabajo a propósito de la explicación en las ciencias sociales, que bien puede leerse como una defensa de cierta estructura narrativa de tales ciencias que nos exige valoraciones muy cercanas a 
las de la literatura para determinar la noción de adecuación de la explicación.

Siendo esas las piezas el objetivo explicito de nuestro juego consiste en mostrar que es posible representarnos la urdimbre de la explicación desde una quiebra de la vieja dicotomfa explicación-comprensión. Quiebra que destacara la presencia de cuando menos una triple clasificación de las explicaciones: causalidad subintencional, intencionalidad y causalidad supraintencional; clasificación con la que se vuelve menos nítido el supuesto espacio peculiar de la comprension. ${ }^{2}$

Pero esa urdimbre no puede permanecer oculta, debemos hacerla explícita si queremos captar su forma particular de articulación con la trama en cuya conjunción se expresa un mundo plausible. La no fijeza, la fluidez de la urdimbre, señala no sólo a dibujos distintos en ciertos detalles sino a cronicas muy diferentes pero plausibles de la práctica social y de su comprensión. Para esta específica zona del trabajo me parecen de enorme importancia las siguientes reflexiones metodológicas del filosofo e historiador de la ciencia antigua, profesor en Cambridge, Gcoffrey Lloyd:

Como helenista, sé lo dificil que nos resulta libramos de las presunciones de que la manera griega de hacer las cosas era el modo natural o inevitable de hacerlas; que la visión griega de las cuestiones a plantear enfocaba justamente aquellas que deben plantearse, las únicas que cuentan; que las preocupaciones de los griegos con sus ideales, modelos y metas, son las preocupaciones que deparan las condiciones necesarias, si no las suficientes, del desarrollo final de la ciencia moderna. Y la mejor manera de desengañarse de esas presunciones es utilizar todos los considerables medios disponibles para la investigación de otros modos de hacer las cosas, otras maneras de percibir las cuestiones clave, otros ideales, otras me- tas, otras preocupaciones. No es tarea fácil pero es posible, ¿La filosofia y la ciencia griegas reflejan caracteristicas fundamentales de la sociedad griega, la política griega, el lenguaje griego, la alfabetización griega, o son reflejo a la vez de las circunstancias económicas, la tecnología o incluso la geografia griegas? $Y$ si es asi, cómo. $Y$ lo mismo, mutatis mutandis, por lo que concierne a China. La unica manera que tenemos de empezar siquiera a poner a prueba la fuerza y la debilidad de cualquiera de estos u otros esquemas explicativos, o de ver qué aspectos se correlacionan con cuáles, consiste en mirar a nuestros colegas del otro lado decididamente más allá de la frontera divisoria -esto es, en mirar más allá del muro institucional que nuestras universidades han levantado como barrera entre nuestras especializaciones [G. Lloyd, material de seminario sobre las teorías de elementos en Grecia y las cuestiones de fundamentación cientifica en China, Madrid 1993, Cambridge 1994].

Uno de los aspectos que trata de poner en juego G. Lloyd en sus estudios de relación entre Grecia y China, en su metodologia de la investigación histórica, aparece como asunto bien diferente de la tradicional búsqueda de similitudes o discrepancias por vía de la comparación. En cierta ocasión, preguntado Lloyd sobre cuál era la razón de su interés por investigar el caso de la ciencia y la filosofia antigua en China, insistió en que la importancia se encuentra en la gran discrepancia, en lo profundamente diferentes a las griegas que son allí no sólo las respuestas que se dan a los problemas sino las cuestiones mismas. Esas reflexiones permiten pensar que Lloyd nos está hablando de crónicas posibles de la historia del pensamiento en Grecia, crónicas que nos podrían acercar a una especifica tradición y a una argumentación no descontextualizada, mediante un esfuerzo de precisión contextual del ámbito discursivo o de la tradición argumental que 
se da en cada caso. En cierta forma se nos propone un análisis en términos de mundos plausibles que aparecen a partir de una comprensión detallada del mundo griego. Aquí lo distintivo es una superación radical de la misma diferencia forma-contenido, de las diferencias académicas entre historia interna e historia externa y de otras grandes dicoto* mías. Un momento decisivo en todo este asunto nos parece que se anuda en torno al problema de la argumentación y al contexto de discusión en el que se produce la construcción teórica.

Queremos pues relacionar esa actitud con la tesis central defendida por G. Hawthorn en Plausible Worlds: ${ }^{3}$ bajo una buena explicacion las posibilidades aumentan en la misma medida en que se reducen. Tanto lo primero que tratamos de explicar cuanto la modelación misma, $y$, hasta la dirección de la explicación que sugerimos, están todas informadas por ciertas figuraciones de lo posible. Algunas de esas configuraciones proceden de otras realidades con las que contrastan. Otras posibilidades no se produjeron. Todas las po* sibilidades que aparecen para un mundo determinado, sean sugeridas por nuestras explicaciones o por contrastes - comparaciones con la que queremos explicar, deberian partir de un mundo tal como aquel que en otros aspectos se produjo. "No se trata de rebobinar el pasado en todo su conjunto." Las consecuencias que trazamos deberian ajustarse con otros procesos que no procedemos a cambiar sino que mantenemos tal como consideramos que marcharon los acontecimientos en ese mundo. Ni los puntos de partida alternativos ni los desarrollos que imputamos deberian parecer fantásticos. Pero la distinción entre lo más o lo menos posible parece simultáneamente demasiado insegura y muy brusca.

Importa observar, siguiendo a Haw- thorn, que las posibilidades que se nos aparecen surgen no sólo a partir de aspectos muy cercanos a los hechos efectivos, a partir de posibilidades que se manifiestan al realizar comparaciones muy cercanas o, en un doble nivel, desde otras posibilidades que fueron examinadas o discutidas en su momento, sino que también ocurre que las teorias mismas son las que nos ofrecen otras posibilidades que no seríamos capaces de ver de otra manera. O lo que es más fuerte, siempre necesitamos de teorías que aparecen como procedimientos explicativos y que nos pueden evitar caer en el simple juego de lo posible; las teorias nos valen así para restríngir ese juego al espacio de lo admisible, lo atendible, lo justificable, es decir, al espacio de lo plausible.

Juguemos, pues, a la búsqueda de un marco té́rico y algo de ello se encuentra en Calvino. El marco teórico aparece como productor de posibilidades y a la vez como constricción de supuestas innứmeras posibilidades; aparece como fuente de plausibilidad.

Estás tratando de leer la última novela de Italo Calvino, Si una noche de invierno un viajero, se te aparecen motivos que te impelen a cierto tipo de reflexión episternológica; uno de los capítulos, ¿quizá mejor habria que decir una de las novelas?, lleva por título "En una red de líneas que se entrelazan", otro u cotra?, "En una red de líneas que se intersecan». La excusa narrativa, que consiste en la figuración de un lector posible que se produce y produce el mismo un conjunto de narraciones posibles, sugiero que parece quedar bien clasificada por el título de este comentario En una red de mundos platusibles, añadiendo o mezclando alguno de los capítulos de Calvino con el título del libro de G. Hawthorn. Obviamente, como siempre ocurre con lo contrafáctico, puede ocur7ir, como vino a suceder en febrero de 
esle mismo año de 1994, que se anuncie una conferencia en Cambridge sobre "Mundos implausibles", pero esto nos sirve sobre todo para recordar que no se trata de una simple combinatoria de las posibilidades. Recuerdas por tanto que la lectura de El vizconde demediado o la de El caballero inexistente, provocaron en su momento cierto tipo de reflexiones sobre la racionalidad y la objetividad en general, pero ahora esos pensamientos aparecen aquí referidos a la obra misma, al trabajo literario mismo. El famoso bucle, el interminable círculo hermenéutico, tratas de cerrarlo, pero se expande en una suerte de loca espiral que te impicle reflexionar aisladamente sobre la verdad. Llegas a pensar que la verdad no es una noción interesante, ¿qué importa quién es el verdadero o el pseudo Cavedagna, personaje de la obra de Calvino?, ¿llega a tener alguna importancia siquiera quién sea el verdadero o el pseudo Italo Calvino? Pasas por fin a la lectura del Calvino de Las ciudades invisibles y ahora ya adquieres una idea especifica, propia, referida a ti mismo sobre el tipo de lectura que quieres realizar; $y$, en la quinta y última de las seis propuestas para el proximo milenio, que Calvino titulo Multiplicidad, y que leiste cuando habias escrito ya buena parte de este comentario, te encuentras no sabes si con la prueba del nueve (en términos de Carlos Pereda) o con la fusión, o quizá con la confusión de horizontes. Quizá hasta cierto grado de confusión nos valga como procedimiento de acercamiento metódico en esta fase postconvencional, Cuando te planteas analizar las relaciones entre Literatura y Filosolía aceptas someterte a un tipo de estudio que te atrae pero del que no sabes muy bien como vas a tratar de salir, incluso cierto miedo escénico se combina con la ansiedad de penetrar en un ámbito del que temes no poder salir. Tratas de resolverlo al modo narrativo.
Recordemos brevemente cierto cuento de Onetti. Un sueño realizado. Alli una mujer trata de exponer la historia de su sueño y pretende contratar a un supuesto director de teatro para conseguir la puesta en escena de su propio sueño e interpretarlo ella misma como actriz. El cuento aparentemente se cierra con la muerte de la misma persona que son̄o el sueño que termina por realizarse. Nos encontramos con que al menos tres de los personajes que aparecen en escena realizan cada uno su propio sueño; lo realizan con ese doble significado del soñar dormido y del soñar despierto que algunos estudiosos de E. Bloch, particularmente de El principio esperanza, han sabido explotar considerablemente con relación a la utopía vertical. ${ }^{4}$ Esos tres sueños configuran además tres particulares historias, tres narraciones bien diferentes aunque integradas en una singular narración por parte de Onetti.

¿Qué nos inquieta, qué puede parecernos sobrecogedor en ese Un stueño realizado de Onetti? Desde mi punto de vista principalmente su peculiar sentido de la objetividad. Un juego de objetividades que se alejan de una simple caracterización de la verdad; aparecen alli las coherencias posicionales, lo adecuado del juego de puntos de vista cuando captas los múltiples puntos de vista. Incluso la forma retórica de la ironía aparece estructurando parte de la combinación de los diversos juegos de lenguaje. ${ }^{5}$ Quizá sea esta la misma posibilidad de la ironía.

Partimos de la convicción de que el juego al que nos somete ítalo Calvino va más alla del juego mismo. Como es sólito en gran parte de sus novelas se nos presenta de forma manifiesta una reflexión múltiple sobre la obra literaria misma, sobre el lenguaje en el que se escribe esa vida literaria y sobre la vida que es escrita por ese lenguaje, 
por tanto resulta que para analizar cuestiones de método es casi trivial partir de esta obra, pretendida superación de la contradicción entre el fragmento y la obra completa, entre la mirada con detalle o el mirar por completo. Mis formas de vida son mis juegos de lenguaje. Pero que decir de esa expresión, acaso son los juegos de lenguaje resultado de mis formas de vida o es que quizá no puedo vivir otra forma de vida que aquella que me posibilitan mis juegos de lenguaje.

En cierto momento el subtítulo de este comentario comenzó siendo «la estructura de la explicación. y ahora hemos adoptado la expresión ala urdimbre de la explicacion». Se trata de sugerir algo menos potente que las formas de práctica tradicional de la filosofia de la ciencia. Cada día se encuentra uno más alejado de quienes ingenuamente pensábamos que existía una interna correlación y casi una simetría entre explicación y predicción. De hecho se suministraba poco más que una técnica de ordenación del conocimiento, desde luego sin ningún substrato ontológico, aunque algunos pretendieran dar el salto mortal de lo metodologico a lo ontológico. Por supuesto que tampoco hace falta dar el salto mortal del individualismo ético al ontologico. En todo caso, en el reverso de la segunda situación, el salto ha de ser moral pese a toda crítica de la falacia naturalista. Lo que quepa decir de lo ontologico no parece que nos venga forzado desde nuestra posición ética o desde nuestra posicion metodológica. Apoyar cierta ontología tras el esquema de explicacion ha sido excesivamente frecuente en la mayor parte de las explicaciones en términos de leyes de cobertura de las que siguen más - menos lejanamente el llamado modelo Hempel-Oppenheim, que en todo caso prefiero llamar Milliano (por $\mathrm{J}$. Stuart Mill). Pero nuestro textil subtitu- lo parece aún exigir más que un primer avance de la relación entre Filosofia y Literatura. ${ }^{6}$

Una primera dirección de la Filosofía a la Literatura, siguiendo algunas indicaciones de Hirschman, nos ha permitido ver que en algunas partes de la obra de Dostoievski, en particular en Memorias del subsuelo, se presentaba la noción de preferencias de segundo orden, concepto importante para analizar y proponer diversos modelos de ser humano sobre los que se construyen las ciencias sociales.

La misma noción de preferencias de orden superior al primero puede servirnos para comprender la noción de regla en el caso de la racionalidad por satisfacción, es decir, en el caso de la racionalidad acotada por ciertas reglas o normas como opuesta a la racionalidad optimizadora o razón olímpica de la elección racional. ${ }^{7}$

Veamos ahora una pequeña historia o al menos la pequeña historia de cómo llegó a mis oídos la crónica de la vida, muerte y posible resurrección de Chocolate, un nunca bien alabado perro de San Luis Potosí. No sabemos muy bien si es perro de San Luis o perro que vivio, padeció y murio en San Luís Potosi. Otro animal de la misma especie, el perro que sobredetermina a Del amor $y$ otros demonios de García Márquez, perro que quizá tenía la rabia, fue mi conducto y quizá mi salvoconducto para conocer esta historia veridica, a diferencia de la historia de su ficción narrada por alguna mujer de edad provecta. ${ }^{s}$ Recuérdese que García Márquez es buen proveedor de salvoconductos en época de leyes Torricelli; así fue, con Márquez. en una de mis manos, cómo alguien que no habia leido esa historia de Márquez me contó su vivencia personal de la historia de Chocolate, un perro de San Luis Potost al que en los años cuarenta le hicieron unas misas gregoria- 
nas a petición de su ama, una mujer de acendrado catolicismo y de muy buena y repleta bolsa cuya última voluntad fue dejar una muy fuerte suma, asaz generosa, para conseguir esas misas por el alma de Chocolate.

Ese mundo de lo real, en el sentido de una objetividad potencial, de una objetividad situacional, aparecía en esa narración como metafórico atractor. Sólo con recordar que el médico farmacéutico judio que se nos presenta en Del amor y otros demonios de García Márquez también aparece deseando ver enterrado su caballo en sagrado, aunque allí se combine con el hecho del ser judío que quizás trataba de ofender a la Santa Inquisición o que acaso quería obtener autodestructivamente el salvoconducto inquisitorial: que era tan religioso que hasta su caballo pretendía enterrar en sagrado. A todo ello unimos que como personaje central de la narración se trasluce también un perro, del que nunca sabemos si adquirió o no la rabia. Todo ese conjunto de acontecimientos produce una construcción permutada que a un observador externo (con cierta teoría adoptada) le provoca un elemento de plausibilidad en ambas narraciones, plausibilidad que aparece como fruto de la situación narrativa. Aparece quizá lo real maravilloso como un elemento que sin duda constriñe el marco de lo plausible pero que expande las posibilidades en la misma medida en que las restrlnge. Repitamos que no consiste en rebobinar el pasado, que no es una simple reconstrucción de todo en todos sus aspectos, se trata por el contrario de hacer cambios aparentemente pequeños que son posibles porque comprendemos bien la situación, la estudiamos en pleno detalle y ese pequeno cambio produce otra crónica posible. Estamos alejados del mundo aleatorio, donde todo es posible; el jardín de nuestros senderos no se bifurca en todos y cada uno de los puntos de nuestra senda.

Esos marcos narrativos nos presentan con intensidad algo a lo que estamos acostumbrados en filosofía contemporánea: el rechazo a los temas básicos o fundacionales en metafísica, en especial el poco interés en una supuesta dualidad entre el mundo externo y nuestras capacidades conceptuales. Como ha señalado recientemente A. Sen," la cuestión puede ser aceptada pero sus implicaciones sólo pueden desarrollarse si reconsideramos el tema mismo de esa dualidad. Por tanto se trata de volver de nuevo al problema de la Objetividad y la Subjetividad.

Así, siguiendo a Sen, diremos que aquello que podemos observar depende de nuestra posición relativa con respecto a los objetos de observacion, que lo que terminamos por creer está influido por lo que observarnos, y, cómo terminamos por actuar está relacionado con nuestras creencias.

Las observaciones, las creencias y las acciones dependientes posicionalmente son centrales para nuestro conocimiento y para nuestra razón práctica. La naturaleza de la objetividad en epistemología, en teoría de la decisión y en ética tiene que tener en cuenta adecuadamente la dependencia paramétrica que respecto a la posición del observador tiene la observación y la inferencia.

Al insistir en la perspectiva posicional se cuestiona la tradición de considerar a la objetividad en la forma de invariancia con respecto a los observadores individuales y a sus posiciones, se cuestiona lo que muchos filosofos han llamado la perspectiva desde ningún lugar (Th. Nagel), es decir, aquella que pretende que una perspectiva de pensamiento es más objetiva cuanto menos descansa sobre la conformación especifica del individuo y de su lugar 
específico en el mundo. Sin duda eso señala hacia algunos aspectos importantes de la concepción clásica de la objetividad pero entra en tensión con la inevitable posicionalidad de las observaciones. Aquí vale la pena señalar una conexión central con todo el pensamiento escéptico tradicional. Desde luego para analizar la conexión no basta con aceptar la Gigura del escepticismo que hemos recibido por parte de la critica de ese pensamiento realizada por los autores del XVIII, Particularmente relevante para nuestro trabajo es el material que se encuentra en Sexto Empírico. Lo que Sexto llama los modos escépticos de argumentar dependientes de quiéri, desde dónde, del objeto, distancia, experiencia anterior, etc., conforman una serie de rasgos cuya importancia no puedo aquí sino mencionar levemente y dejarlos para otra ocasión. Tienen importancia para diversas discusiones epistemologicas contemporáneas; obviamente para el rancio tema del realismo y las diversas variedades de epistemologías naturalizadas. $^{10}$

Si tenemos en cuenta, además, que nosotros no somos un simple dato paramétrico, que no somos un dato fijo o permanente aunque sea intercambiable, tenemos que incorporar lo que podemos llamar una dependencia estrategica de la objetividad y conectarla con una interacción estratégica de la percepción del otro. El contenido de una afirmación objetiva puede bien ser la manera en que un objeto aparece desde una posición específica de observacion. Desde luego esto es puro Sexto Empírico.

La objetividad de las observaciones viene a ser una caracteristica dependiente de la posición. No es tanto una perspectiva desde ningún lugar, una visión desde ningún sitio [a view from nowhere], sino más bien una que se configura desde alguin determinado lugar [from a delineated somewhere] [A. Sen, op. cit.].

Me parece que la posición defendida por Sen junto a una adecuada reconceptualización de la herencia escéptica puede ayudarnos al estudio de las condiciones mínimas para que se produzca una argumentación "compartida", para la delimitación de unas condiciones epistémicas compartidas que vendrán a reflejar un entrenamiento similar, una educación artística parecida. ciertos intereses comunes, etc., que ayudan a comprender el punto de vista narrativo. La dependencia de la posición no se aplica exclusivamente a las afirmaciones observacionales directas. La tensión es mucho más amplia porque también es relevante para las decisiones sobre las creencias y las acciones.

Todos los componentes anteriores conducen, al menos así me lo parece, al expediente analítico de los mundos plausibles y a su conexión con el análisis contrafáctico. Una primitiva idea de los contrafácticos aparecía en el campo de la filosofía de la historia y, como sabenos, se trataba de la paradoja provocada por aguellos condicionales cuyo antecedente está contra los hechos (a partir de la falsedad del antecedente obtenemos que, sea cualquiera que sea el consecuente, el condicional considerado como bloque, como oración, resulta ser verdadero). Muchos fueron los debates producidos por ese tipo de argumento, pero el punto que nos reclama la narración de Chocolate, sobre todo la narración oral, aún más que la versión de García Márquez, es que nos coloquemos en alguna posición, no tanto que nos pretendamos ciudadanos de un mundo universal, abstracto. Aquella supuesta neutral posición de panóptico que no 
está en ninguna parte, y que por ello se pretende en todas, que pretende ofrecer una interpretación, "la interpretación», ajustada, correcla, fiable, verdadera de esa narración, no es sino el viejo mito de la referencialidad como externa a nuestra construcción de sentido. Por el contrario, la narración nos pide, nos reclama, que nos coloquemos exactamente en algún punto concreto, que nos identifiquemos con alguien, sea de la narración sea como lector de la narración (como en Calvino); pero que nos coloquemos en algún punto para poder enconirar la objetividad que se obtiene desde esa posición, como parámetro posicional del espacio de la construcción del sentido.

El espacio privilegiado para la filosofia desde la literatura es asi la posibilidad de construcción de un mundo plausible que nos hace ver el camino plural de la individualidad. En definitiva las ciencias sociales mismas aparecen como una forma de narrativa: la selección de los datos y la supuesta descripción de ellos corresponden a una forma particular de narrativa desde la que se han seleccionado las categorías, los conceptos teoricos que nos permiten la selección de ciertos datos. Esos conceptos actúan como filtros informativos que nos producen una seleccion, dejan pasar ciertas cosas e impiden que otras pasen. Toda red conceptual actúa así como un filtro, mejor aún como una especie de membrana semipermeable que construye, elabora, da sentido a nuestra descripción y facilita el nuevo contenido semántico de la metáfora, anula la idea mecánica de la metáfora como simple reescritura, como reductíble a la expresión del significado literal. Nos dota de nuevo sentido.

Para completar nuestra perspectiva seria preciso analizar las nociones de racionalidad y de información, y señalar algunas conexiones entre este tipo de planteamientos y otros procedentes de problemas genéricamente epistemológicos. Asf, por ejemplo, algunas formas de razonamiento, particularmente las caracterizadas como inductivas, se han relacionado a veces con nociones procedentes de la teoria de juegos. Las conexiones no fueron más allá de relacionar esa estrategia con las diversas nociones de equilibrio en los juegos de estrategia $y$, en definitiva, se desarrollaron principalmente por vía de la teoría de la inferencia estadística y con una perspectiva estática que discutia el equilibrio del juego sin tener en cuenta el proceso de equilibración mediante el cual se supone que se llega al equilibrio. Quizá esa orientación estática era la que hacía poco interesante para los problemas epistemologicos el uso de la teoría de juegos, y por ello se produjo cierta reducción de su uso para la reflexión sobre temas epistemologicos.

Sin embargo, ciertos desarrollos recientes en la teoría de juegos, junto a la renovación de la discusión episte. mologica que se apoya en la teoría de la decisión, permiten pensar en una reconsideración de temas tradicionalmente epistemológicos desde esta perspectiva. En particular los trabajos que se vienen haciendo, sobre la propia fundamentación de la teoría de juegos (Ken Binmore), sobre elección dinámica y sobre deliberación racional, son todos materiales que permiten cierta reconsideración de la noción de argumentación desde la teoría de juegos y, como elemento derivado, ofrecen al menos una nueva forma de hablar, cuando no una nueva perspectiva, sobre temas como el de la inducción, la explicación o la causalidad.

Si no queremos reducir la argumentación a una presentación sintética de lo que ya es nuestro conocimiento. 
tendremos que fijarnos en que durante el proceso argumentativo se produce un cambio de nuestra situación cognitiva, de nuestros estados de creencias, de manera que los compromisos que establecemos con nuestro conocimiento han cambiado; concluida la argumentacion estamos, sea real o pretendidamente, en un estado cognitivo diferente, y precisamente en un nuevo estado donde se ha incorporado la conclusión del argumento como parte de nuestro nuevo estado de creencias.

El análisis de la argumentación creemos que puede verse enriquecido por las aportaciones procedentes de los estudios sobre la deliberación racional, la elección en situaciones de conflicto irresuel to y la dinámica de los estados epistémicos. Si los individuos tienen que iniciar el debate desde un conocimiento común compartido por lo que se refiere a su propia racionalidad, y constituida la racionalidad exclusivamente por la consistencia de la elección, la ausencia de análisis sobre la génesis de las propias creencias hacía que, salvo que estuviésemos en un universo complctamente cerrado, fuese imposible comprender el acceso al equilibrio. Por tanto, una primera cuestion de método es que tendremos que intentar modelar el juego de la argumentación en forma extensa, no matricial, de manera que se puedan captar mejor los procesos dinámicos de la argumentación. La presentación extensional pretende representar las transformaciones de nuestros estados de conocimiento en las diversas fases de la argumentacion, mediante lo que podremos llamar transiciones de estado de conocimiento. Precisamente la atención a la conformación de las creencias de las que partimos, la reflexión sobre el software que aplicamos y sus posibles transformaciones a medida que avanza el proceso deliberativo nos hace decir que cualesquiera que sean los problemas de la inducción cientifica seguramente están representados por algo más que por la "trivial manipulación algebraica llamada regla de Bayes" (K. Binmore).

Cuando parece interesante proseguir una investigación y desarrollar uma argumentación es cuando no conocemos el resultado. Buscamos algo inesperado que suministrará cierto grado de sorpresa; si se tiene de antemano el resultado no parece razonable argumentar. Ni siquiera en la sistematización deductiva está ausente ese grado de sorpresa, de obtención de lo inesperado. Hasta en la demostración más simple se olvida momentáneamente la verdad de la consecuencia para poder «obtenerla». La argumentación surge en un terreno parecido a aquel en el que según Dewey (I. Levi, Hard Choices) se da la necesidad de la teoria moral; es necesaria cuando hay conflicto entre valores: si conocemos el bien no tenemos nada que analizar sólo nos queda practicarlo; cuando aparece un conflicto entre valores es cuando se precisa la investigación moral.

En la argumentacion parece imprescindible introducir un elemento dinámico, fluido, que no se detecta en la forma estándar de la explicación. A pesar de su trivialidad es preciso atender a dos cuestiones importantes en la argumentación: por un lado tenemos los puntos de partida y de arribada de la argumentación, pero de otra parte debemos fijar también nuestra atención en el camino mismo. La tendencia dominante ha sido la de considerar el discurrir de la argumentación como un simple mecanismo regulado; sin embargo es muy importante observar la argumentación misma como productora de información para su propio despliegue. La argumentación no es una senda preparada de antemano 
para que discurramos por ella, no es tanto una trama fija que da estructura a los pasos argumentales cuanto la urdimbre que se va configurando en el proceso mismo de la práctica deliberativa. Desde luego hay argumentaciones que no admitiremos como explicaciones, por ejemplo aquellas que facilitan la aceptación de un resultado y nos comprometen con la crónica sugerida pero que no incrementan la probabili- dad de la ocurrencia de ese acontecimiento; quizá como candidatos a explicación tengamos que pensar en algún subconjunto propio del mundo de la argumentación. Qué tipo de argumentaciones se aceptarán como explicaciones es un problema posterior que por ahora no abordaremos. Esperemos que con todo ello estemos a las puertas de una reflexion sobre la urdimbre de la explicación.

\section{NOTAS}

1. Se presenta una versión sintetizada de materiales que se han discutido en una reunion del Departamento de Crítica Literaria de la UAM-I, en el proyecto *Filosofia y Literatura* del Instituto de Investigaciones Filosoficas de la UNAM y en el proyecto homónimo del CSIC dirigito por Carlos Thiebaut y Jose $M$. González. Los comentarios y criticas de Aralia Lobez, Rocio Antúnez, Marfa Herrera, Carlos Pereda, Jesusa Rodríguez, Mariflor Aguilar. Griselda Gutierrez, Ivy Boreal desde un lado del Atlántico, de José M. González, Jose M. Marinas, Javier Muguerza, Carlos Thiebaut desde el otro, junto a las opiniones de otros participantes o lectores, han sido muy importantes para corregir aspectos, aunque impenitentemente continuo en el error.

2. Permeando todo el texto aparece la tensión entre epistemologins de corte tradicional y algunas versiones que podriamos clasificar como epistemologías radicales, que van desde las realizadas desde una optica feminista a las que proceden de versiones que podremos llamar no fundacionalistas y paramétricas. En este paso nos hacemos eco de la conocida propuesta de J. Elster aparecida, por ejemplo, en Explaining Technical Charge, propuesta que por mi parte he analizado en varias ocasiones, sin ir más lejos en Isegoría, n." 3 .

3. El libro publicado originalmente en $1992 \mathrm{ha}$ sido muy bien acogido, se ha agotado ya la tercera edición (en dos años) y está en prensa la versión en español (también en Cambridge Univetsity Press) Iraducida por Gloria Camevali.

4. Propuesta avanzada y defendida por J. Muguerza, como distinción pero tambien como defensa de Bloch, en aRazón, Utopía y Disutopia. Desde la perpleitadad, Madrid, FCE, 1990 , pp. 377. 419.

5. Cuestion que discuti ampliamente con Rocío Antúnez, profesora del área de Literatura de la UAM, México, quien llamó mi atención hacia el relato de Onetti.
6. Relación cuya relevancia metodológica traté de analizar en $i$ Es inteligente ser racional?*, Sistenn (1993).

7. Razón olímpica que claramente tiene muchos parecidos con la razón austera criticada por Pereda. En el capítulo 10 de la segunda parte del libro de Carlos Pereda, Razbin e incertidumbre, México D.F., Siglo XXI, 1994, a propósito de la posición de Wittgenstein sobre los objetos matematicos se sugiere un camino, el de la fuerza del mito platónico, que pienso puede apa* recer como esa capacidad resolutiva que dan las reglas inismas en la acción por satisfacción. Se da ahr un paso más en la elaboración de su concepto de razón enfática, aún excesivamente formalista, a la que le encuentro alguna correspondencia con $\mathrm{mi}$ propia propuesta de racionalidad imperfecta.

8. Doña Jesusa Rodríguez, quien me contó la historia y a quien aún no he pedido autorización para contar la bistoria que estructura esta parte de mi trabajo, es una alegre y locuaz mujer en su primera ancianidad, con enorme fuerza narrativa y que encierra en si misma la virtud de la plausibilidad. En conversacion con ella comprendi una posible interpretacion de la nocion de punto de vista narrativo, una razón más por la que le estoy muy agradecida.

9. En Philosophy and Public Aftairs, vol, 22, 2 (primavera 1993), pp. 126-145.

10. Parte de la relevancia he tenido la suerte de discutirla con Gloria Camevali (Clare Hall, Cambridge) a propósito de su importante investigación en marcha sobre el espacio y la pintura abstracta del $\mathrm{xx}$. El estudio en detalle de la obra de Sexto Empírico lo debo principalmente a una sugerencia de G. Lloyd y al trabajo en el Sunday Evening Seninar de Cambridge junto a uno de sus inspiradores, Ultich Timpte, quien ayudó mucho con sus agudas reflexiones y facilito la tarea para poder avanzar en el texto griego. 